Published in final edited form as:

Neurology. 2007 October 30; 69(18): 1789-1799. doi:10.1212/01.WNL.0000287431.88658.8b.

\title{
Updated research nosology for HIV-associated neurocognitive disorders
}

\begin{abstract}
A. Antinori, MD, G. Arendt, MD, J.T. Becker, PhD, B.J. Brew, MBBS, MD, FRACP, D.A. Byrd, PhD, M. Cherner, PhD, D.B. Clifford, MD, P. Cinque, MD, PhD, L.G. Epstein, MD, K. Goodkin, MD, PhD, M. Gisslen, MD, PhD, I. Grant, MD, R.K. Heaton, PhD, J. Joseph, PhD, K. Marder, MD, MPH, C.M. Marra, MD, J.C. McArthur, MBBS, MPH, M. Nunn, PhD, R.W. Price, MD, L. Pulliam, PhD, K.R. Robertson, PhD, N. Sacktor, MD, V. Valcour, MD, and V.E. Wojna, MD

Clinical Department (A.A.), National Institute for Infectious Diseases "Lazzaro Spallanzani," Instituto di Ricovero e Cura a Carattere Scientifico (IRCCS), Rome, Italy; Department of Neurology (G.A.), Heinrich Heine University, Dusseldorf, Germany; Departments of Neurology and Psychiatry (J.T.B.), University of Pittsburgh, PA; Department of Neurology and HIV Medicine (B.J.B.), St. Vincents Hospital, University of New South Wales, Sydney, Australia; Department of Psychiatry and Pathology (D.A.B.), The Mount Sinai Medical Center, New York, NY; Department of Psychiatry (M.C., I.G., R.K.H., K.R.R.), University of California, San Diego; Department of Neurology (D.B.C.), Washington University, St. Louis, MO; Clinic of Infectious Diseases (P.C.), San Raffaele Hospital, Milan, Italy; Department of Neurology (L.G.E.), Children's Memorial Hospital, Chicago, IL; Departments of Psychiatry, Neurology and Psychology (K.G.), University of Miami, FL; Department of Infectious Diseases (M.G.), Sahlgrenska University Hospital, Göteborg, Sweden; Center for Mental Health Research on AIDS (J.J.), National Institute of Mental Health, Bethesda, MD; Department of Neurology (K.M.), Columbia University, New York, NY; Department of Medicine, Allergy and Infectious Diseases (C.M.M.), Department of Neurology, University of Washington Seattle; Departments of Neurology, Pathology, and Epidemiology (J.C.M.), and Department of Neurology (N.S.), Johns Hopkins University, Baltimore, MD; National Institute of Neurological Disorders and Stroke (M.N.), NIH, Bethesda, MD; Departments of Neurology (R.W.P.) and Laboratory Medicine and Medicine (L.P.), University of California, San Francisco; Department of Neurology (K.R.R.), University of North Carolina School of Medicine; Hawaii AIDS Clinical Research Program (V.V.), University of Hawaii, Honolulu; and NeuroAIDS Program and Department of Neurology (V.E.W.), University of Puerto Rico, Medical Sciences Campus
\end{abstract}

\section{Abstract}

Copyright $@ 2007$ by AAN Enterprises, Inc.

Address correspondence and reprint requests to Dr. Justin C. McArthur, Johns Hopkins Hospital, Meyer Building, Room 6-109, 600 North Wolfe Street, Baltimore, MD. 21287-7609; jm@jhmi.edu.

Supplemental data at www.neurology.org

Disclosure: The authors report no conflicts of interest.

Disclaimer: This work was written as part of Dr. Jeymohan Joseph's and Dr. Mike Nunn's official duties as government employees. The views expressed in this article do not necessarily represent the views of the NIMH, NINDS, NIH, DHHS, or the United States government. 
In 1991, the AIDS Task Force of the American Academy of Neurology published nomenclature and research case definitions to guide the diagnosis of neurologic manifestations of HIV-1 infection. Now, 16 years later, the National Institute of Mental Health and the National Institute of Neurological Diseases and Stroke have charged a working group to critically review the adequacy and utility of these definitional criteria and to identify aspects that require updating. This report represents a majority view, and unanimity was not reached on all points. It reviews our collective experience with HIV-associated neurocognitive disorders (HAND), particularly since the advent of highly active antiretroviral treatment, and their definitional criteria; discusses the impact of comorbidities; and suggests inclusion of the term asymptomatic neurocognitive impairment to categorize individuals with subclinical impairment. An algorithm is proposed to assist in standardized diagnostic classification of HAND.

In this report, we describe the existing American Academy of Neurology (AAN) criteria, ${ }^{1}$ and discuss suggested areas for revision based on our experience, particularly in the decade since highly active antiretroviral treatment (HAART) became widely available in the developed world. Changes to the diagnostic criteria for HIV-associated myelopathy and sensory neuropathies were not discussed. We also present a view of the changing face of HIV-associated neurocognitive disorders (HAND), specifically how the temporal progression may have been modified by HAART. An algorithm is presented, which we believe to be a useful operational tool for diagnosis and identification of HAND. Although these proposed criteria are based on more extensive research experience than was available when the AAN criteria were originally proposed, it is recommended that these be regarded as research criteria that will require further study before they are definitively adopted into clinical practice.

\section{SECTION 1: REVIEW OF DEFINITIONAL CRITERIA}

\section{A: Current AAN definitional criteria}

The 1991 AAN criteria defined two levels of neurologic manifestations of HIV infection: HIV-associated dementia (HAD) and minor cognitive motor disorder (MCMD) (table E-1 on the Neurology ${ }^{\circledR}$ Web site at www.neurology.org). Briefly, the AAN criteria for HAD were 1) an acquired abnormality in at least two cognitive (nonmotor) areas causing impairment in work or activities of daily living (ADLs), and 2) either an abnormality of motor function or specified neuropsychiatric or psychosocial functions (e.g., motivation, emotional control, social behavior). Moreover, the patient had to have sufficient consciousness for cognitive abilities to be assessed, and could not have other etiologies that might explain the disorder. The AAN diagnostic scheme defined three subtypes of HAD:

1. HAD with motor symptoms (criterion 1 met fully, but only motor symptoms meeting criterion 2)

2. HAD with behavioral or psychosocial symptoms (criterion 1 met fully, but only behavioral symptoms meeting criterion 2)

3. HAD with both motor and behavioral/psychosocial symptoms (criteria 1 and 2 met fully) 
The AAN criteria also defined a less severe condition called MCMD. The essential features of MCMD according to the AAN criteria were a history of impaired cognitive/behavioral function in two areas (e.g., impaired attention-concentration, mental slowing, abnormal memory or other cognitive functions, slowed movements, incoordination, personality change, irritability, lability), and these abnormalities cause mild impairment in work or ADLs, do not meet criteria for HAD or HIV-associated myelopathy, and cannot be attributed to other etiologies.

In our review, we identified issues that may restrict the applicability of the 1991 AAN criteria. One issue is that the number of domains of impairment that should be examined for diagnosis was not clearly defined. Moreover, the degree of neurocognitive impairment was not fully specified, permitting variability in the clinical estimation of severity. Third, there appeared to be some overlap between the criteria for HAD with mild functional decline and MCMD. Finally, the schema did not admit mild forms of reliably identified cognitive difficulties which had not developed to the point of interfering substantially with everyday functioning. There is increasing recognition of the frequency of confounding conditions that are potentially acting as compounding factors (deficits with mixed etiologies), and these were not adequately considered in the 1991 schema other than the simple exclusionary stipulation.

\section{B: Proposed research criteria developed by HIV Neurobehavioral Research Center at UCSD}

To address some of these concerns, the HIV Neurobehavioral Research Center (HNRC) at UCSD established working research criteria for HIV-related neurocognitive complications which were intended to represent a refinement of the AAN criteria. These criteria recognize the following three conditions: asymptomatic neurocognitive impairment (ANI), HIVassociated mild neurocognitive disorder (MND), and HIV-associated dementia (HAD) (table).

These modified criteria were developed by starting with the existing AAN criteria, and introduced changes based on research and observations made at HNRC, and other published sources. The most notable change is addition of the category of ANI based on the observation that some individuals have demonstrable (and usually mild) cognitive impairment demonstrated by formal neuropsychological tests without any observed abnormality in everyday functioning. The caveat to this statement is that the assessment of functional capacity is difficult and frequently requires third-party report, or prolonged observation. Furthermore, the HNRC criteria are more fully specified in terms of types and severities of cognitive difficulties.

ANI is defined by performance at least $1 \mathrm{SD}$ below the mean of demographically adjusted normative scores in at least two cognitive areas (attention-information processing, language, abstraction-executive, complex perceptual motor skills, memory, including learning and recall, simple motor skills or sensory perceptual abilities); these criteria specify that at least five cognitive domains be examined or observed. Finally, the impairment does not occur solely as part of a delirium (i.e., a confusional state secondary to opportunistic CNS disease, vascular insult, metabolic derangement, drug effects, or other systemic disorders) and, as in 
all AAN criteria, the diagnosis is possible only if the cognitive impairment cannot be explained by other comorbidities. There does appear to be empirical support to adding this third category of HIV-related neurocognitive disorder, because it appears to have a priori prognostic value. $^{2}$

The MND defined by HNRC is similar to the MCMD previously defined by AAN but, in addition to criteria for asymptomatic neurocognitive abnormality, MND requires that there also be impairment in everyday functioning. Specifically, MND is defined by the following features: 1) an acquired mild-to-moderate impairment in cognitive function documented by a score of at least 1 SD below demographically corrected norms on tests of at least two different cognitive domains, 2) the cognitive impairment interferes, at least mildly, with activities of daily living, 3) the impairment does not meet criteria for delirium or dementia, and 4) the impairment is not fully explained by comorbid conditions.

Finally, diagnosis of HAD according to these suggested criteria requires 1) acquired moderate-to-severe cognitive impairment, documented by a score at least 2 SD below demographically corrected normative means in at least two different cognitive areas, 2) marked difficulty in ADLs due to the cognitive impairment, 3) the impairment does not meet criteria for delirium, and 4) the impairment is not adequately explained by comorbid conditions. (Note that there is an expanded discussion of comorbid conditions, and how to interpret their possible impact in the context of HAND, on the Neurology ${ }^{\circledR}$ Web site.)

\section{C: Comparison of updated and existing definitional criteria}

A fundamental aspect of the updated definitional criteria for HIV-associated neurocognitive complications is the greater priority given to the cognitive aspects of impairment compared to motor, social/personality, or emotional difficulties.

To determine if giving more weight to the cognitive area had validity, Cherner et al. ${ }^{2,3}$ examined the correspondence of AAN and HNRC diagnoses to neuropathologic outcome, i.e., presence of HIV encephalitis at autopsy. When the two sets of definitional criteria were compared regarding the classification of patients as either neurocognitively normal or impaired before death, the agreement was $79 \%$ (31 of 39 patients). However, when specific diagnoses were compared, the two sets of criteria gave consistent diagnoses only for 21 patients $(54 \%)$. When pathologic evidence of HIV encephalitis was considered as the gold standard, 25 patients were correctly classified by AAN criteria as having neurocognitive involvement, compared to 28 patients correctly classified by HNRC criteria. In particular, 4 patients with HIV encephalitis were considered neurocognitively normal by AAN criteria, while 1 patient with encephalitis was considered normal by HNRC criteria; 8 patients with HIV encephalitis were classified as neurocognitively normal by both sets of criteria. Thus, both sets of definitional criteria were reasonably accurate in predicting autopsy diagnoses of HIV encephalitis. However, the HNRC criteria were slightly better in terms of positive predictive power (95\% vs $88 \%$ ), sensitivity ( $67 \%$ vs $56 \%$ ), and specificity (92\% vs $83 \%$ ), possibly due to the inclusion of a third, asymptomatic neurocognitive condition.

Another study, carried out by Wojna and colleagues in San Juan, Puerto Rico, compared the diagnoses of cognitive impairment in HIV-infected women using several sets of criteria, 
including the standard AAN criteria as well as AAN criteria modified to include a class of impairment without functional decline in ADLs (similar to the HNRC asymptomatic category). ${ }^{4}$ By standard AAN criteria, 53.6\% of subjects were considered neurocognitively normal, $18.8 \%$ had a diagnosis of MCMD, and 23.2\% had HAD. Modification of the AAN criteria to include asymptomatic neurocognitive abnormality had a notable effect: $31.1 \%$ of cases were cognitively normal, $20.3 \%$ had ANI, $18.8 \%$ had MCMD, and $23.2 \%$ were diagnosed with HAD. Thus, the modified criteria helped investigators distinguish a subgroup of HIV-infected patients with asymptomatic neurocognitive impairment (more than one-third of those initially considered normal). The National NeuroAIDS Tissue Consortium has utilized a subsyndromic category in their neuropsychologic assessments since 1999; as of August 2006, 14\% of 1,328 advanced-stage HIV-infected individuals had diagnoses of subsyndromic impairment at their baseline evaluation (Dr. Susan Morgello, personal communication, 2007).

\section{CONCLUSIONS: SECTION 1}

The existing AAN criteria have served researchers and clinicians well for 15 years. They offer reasonable sensitivity and specificity for predicting future neuropathologic diagnoses of HIV encephalitis, although the positive predictive power can be enhanced by considering asymptomatic neurocognitive abnormality. A limitation of the existing AAN criteria is that they do not recognize a subgroup of HIV-infected patients $(<15 \%)$ who actually have neurocognitive impairment despite the absence of overt functional decline in ADLs. We recognize that further work needs to be conducted on the real-life impact of ANI, but at this stage recommend adding this condition to the criteria for HAND. We recommend that the presence and degree of neurocognitive impairment constitute the fundamental criteria for establishing a diagnosis, while other criteria, e.g., motor disorders and emotional or personality changes, be considered ancillary or corroborative information, possibly for defining disorder subtypes. Finally, determination of neurocognitive impairment should be based on appropriately normed tests (see the Neurology ${ }^{\circledR}$ Web site), and should consider the presence of confounding factors. It is timely to work toward a revision of the diagnostic criteria along the lines displayed in the table, and we strongly recommend that revised criteria be field tested and further refined through research.

The area of quantitative testing of motor function needs further exploration, but data from Arendt et al. ${ }^{5}$ seem promising in that relatively simple quantitative measures seem to track with cognitive improvements associated with HAART. The predictive value of quantitative motor test abnormalities for evolving cognitive impairment needs further delineation and field testing.

\section{SECTION 2: CHANGES IN THE SYNDROMIC NATURE OF HIV-ASSOCIATED NEUROCOGNITIVE DISBURBANCES}

There have been substantial changes to HIV disease with the introduction of HAART which have affected cognitive impairment in a number of areas. ${ }^{6}$ Such changes impact on the recognition of the disorder and raise questions relating to its fundamental nature. These encompass the areas of epidemiology, natural history, clinical phenotype, and confounding 
conditions. We have also observed that a substantial proportion of HIV-infected persons $(<20 \%)$ have bidirectional changes in neurocognitive symptomatology, fluctuating from normal to abnormal and vice versa, at different levels of severity. Any revised nosology should recognize this fluctuating category, while research should try to understand the causes of this fluctuation and the effects on treatment outcomes, everyday functioning, and patient survival. The inherent variability of neuropsychological testing needs to be taken into account in the interpretation of these fluctuations.

\section{A: Time course and stability of neurocognitive impairment}

The time course and stability of neurocognitive impairment in HIV/AIDS over time have been explored using data from several cohorts. For example, an HNRC cohort that included 534 HIV-seropositive and 141 seronegative persons showed that at baseline, 14\% of HIVnegative persons were cognitively impaired (at any level), as were $27 \%, 44 \%$, and $52 \%$ of subjects with CDC stages A, B, and C disease (figure 1). Over time, considering only HIVinfected persons, $47 \%$ remained cognitively normal and $11 \%$ remained impaired; furthermore, $18 \%$ improved and stayed improved (stably improved), $4 \%$ worsened and remained so (stably declined), and 19\% fluctuated between impaired and normal at the different examinations. Data from demographically matched seronegative controls tested repeatedly $(\mathrm{n}=30)$ showed that $80 \%$ remained cognitively normal, $3 \%$ were stably impaired, $7 \%$ improved, $3 \%$ worsened, and $7 \%$ fluctuated. To address the possibility that changes in neurocognitive classification might reflect error variance, the rates of changed vs unchanged classifications were compared between groups. The proportion of HIV+ who changed $(42 \%)$ was greater than that for HIV- $(17 \%)\left(\chi^{2}=7.629 ; d f=1 ; p<0.01\right)$, suggesting that the frequency of neurocognitive change in HIV+ was unlikely to be due to chance. These data are summarized in figure 2 .

Diagnostic transitions over 1 year were also studied by Valcour and colleagues in the Hawaii Aging with HIV cohort, with similar findings (V. Valcour, personal communication, 2005). Of 37 patients who at baseline were neurocognitively normal, at 1 year $30 \%$ had progressed to some stage of impairment. Of 53 patients initially diagnosed with asymptomatic neurocognitive abnormality (neurocognitively abnormal without functional decline), $17.7 \%$ were classified as normal 1 year later, while $44.1 \%$ had progressed to more severe categories of impairment. This pattern was repeated for other patients with baseline diagnoses of MCMD and HAD, although the proportions of patients improving 1 year later (receiving less severe diagnoses or considered cognitively normal) reduced as the initial severity of the disease increased (figure 3).

Finally, similar observations were made by the Northeast AIDS Dementia Consortium. In particular, $21 \%$ of subjects initially diagnosed with MCMD became neurocognitively normal at follow-up, while $23 \%$ of those considered normal at study entry were diagnosed with MCMD at follow-up. The degree of fluctuation observed in these cases of changed diagnostic category was relatively small; i.e., gross changes in levels of functioning were not seen.

Thus, observations from at least three separate cohorts suggest that a sizable proportion of patients have a fluctuating course of neurocognitive impairment over time, and that 
normalization of symptoms is possible. For this reason, the qualifier "in remission" is proposed for the three research criterion sets displayed in the table. It remains to be determined whether these transitions reflect biologic changes induced by responses (or failures) of antiretroviral therapy. Similar observations have been noted in other cohorts, and appear to be independent of plasma viral load and switches in $\operatorname{HAART}^{7}$ (J.C. McArthur, personal observation, 2006). These fluctuations in the cognitive state have parallels with mild cognitive impairment, a disorder commonly observed in the elderly, and the cognitive fluctuations that are also observed in relapsing-remitting multiple sclerosis.

\section{B: Changes in the temporal progression of HIV-associated neurocognitive disorders with HAART}

HAND remains frequent even in the era of HAART. Epidemiologically there has been a significant decrease in the incidence of the most severe manifestation of HAND (i.e., HAD) but in most studies this has been counterbalanced by an increase in the prevalence. ${ }^{7,8}$ However, the epidemiologic aspects of less severe forms of HAND are much less well defined, although informally most investigators consider both incidence and prevalence to be increasing. Recognition of HAD also requires understanding that it is somewhat different to its counterpart in the pre-HAART era, and is perhaps evolving. For example, in the preHAART era HAD almost exclusively occurred in those with CD4 cell counts below 200. Now patients who develop the disorder on HAART may have normal or near-normal CD4 cell counts. ${ }^{9}$ The plasma HIV viral load, which was always elevated and often markedly so in those with HAD, is usually now less elevated and very occasionally may be below the detection limit. HIV neurocognitive disorders are now milder and survival is considerably longer. Nonetheless, they can still impact upon quality of life and optimal medical management. HIV neurocognitive disorders in the HAART era may occur even in those patients who do not have other evidence of active HIV disease. The latter is important to recognize as no current therapies would be expected to produce neurologic improvement when there is no active viral replication. ${ }^{10}$

HIV neurocognitive disorders may be evolving in terms of their associated features. In the pre HAART era, the confounding conditions were largely related to opportunistic complications. Now with the higher CD4 cell counts there are new factors such as the effects of hepatitis $\mathrm{C}$, increased age and associated conditions such as HAART-related hyperlipidemia, hypertension, and possibly Alzheimer disease. These confounds may also interact with HIV's effect on the brain, leading to a compounding of the deficit.

To assist clinicians in diagnosing the disorder and provide a framework that allows these issues to be addressed, we propose changes to the definition of the disorder and introduce criteria that allow qualification of the progression of HAND according to its activity.

\section{C: Importance of other confounding conditions in association with HAND}

It is clear that HIV-infected patients are potentially vulnerable to cognitive effects from other conditions. While these can be confounds to the accurate diagnosis of HAND, they can also have a compounding effect if HAND is already present. The word "confound" has the connotation of an alternate diagnosis, not necessarily an additional diagnosis. It is important 
for clinicians to realize that confounding conditions may also be acting as compounding conditions (table E-4). As such, treatment for HAND needs to be considered in addition to treatment of the confounding condition. There are, of course, numerous conditions which might confound the accurate diagnosis of ANI, MND, or HAD. Rather than listing all, we will focus on those that the group believed were most relevant.

\section{D: Importance of demographic adjustments in interpreting neurocognitive test results}

An important issue regarding the use of neurocognitive tests is that they be appropriately normed for the study population. In particular, performance on these tests is subject to influence by age, education level, ethnic or racial background, and gender.

Byrd and colleagues (New York, NY) studied the effect of ethnic background on the apparent prevalence of HIV-associated neurocognitive disorders in an urban cohort of advanced HIV-infected adults using the standard AAN criteria. Results indicate that using standard AAN criteria, which are primarily based upon data from white normal subjects, over $90 \%$ of HIV-infected minorities were diagnosed with HAD/MCMD. In contrast, only about $78 \%$ of the white population was considered impaired. Heaton and colleagues made similar observations at the HNRC: using standard test norms to rate a group of healthy uninfected subjects, $33 \%$ of African Americans and 15\% of white subjects were considered cognitively impaired. When African Americans were scored using norms derived from a large group of healthy African Americans, the percentage of impaired subjects was reduced to $19 \%$, indistinguishable from that for white subjects. Regarding HIV-seropositive subjects, cognitive impairment among white subjects was 38\%, while that for African Americans was $71 \%$ using standard norms, but $44 \%$ using culturally adapted norms (figure 4). Finally, Cherner and colleagues at HNRC administered the figure learning test to healthy Spanishspeaking persons: using standard norms $31 \%$ were considered to be cognitively impaired, but with norms designed for Spanish-speaking persons only 15\% were classified as impaired (similar to the rate in a majority population of HIV- native English speakers). Cherner and colleagues also demonstrated the effects of education on neuropsychological test results: $40 \%$ of persons with 1 to 5 years of education were classified as impaired on the figure learning test, but when education-adjusted norms were applied, the rate of impaired persons dropped to under $20 \%$.

\section{SUMMARY: SECTION 2}

The concept of HIV-associated neurocognitive disorders has evolved since the original descriptions of HAD in the early years of the epidemic. Antiretroviral therapy has improved longevity substantially, and also may reverse neurocognitive deficits in many cases. One obvious impact of recognizing the term of ANI and incorporating it into clinical practice and research usage would be to encourage more frequent neurologic follow-up for individuals with ANI, to detect early functional impairments and potential transition to MND or HAD. In addition, the recognition of ANI might promote the initiation of antiretroviral therapy, independent of CD4 count or plasma HIV RNA levels. These issues/questions are unresolved and require future study. 


\section{SECTION 3: PROPOSED ALGORITHM FOR CLASSIFYING HAND}

The three conditions comprising HAND—ANI, MND, and HAD—may be classified using a variety of specific clinical and laboratory-based methods, depending upon the resources available in the setting where the patients are being evaluated. Nevertheless, standardized procedures should be followed whenever possible, both to collect the needed information and to interpret that information to make three types of determination: 1) the presence and severity of neurocognitive impairment, 2) the presence and severity of functional decline, and 3) the degree to which cognitive impairment or functional decline are likely to have been influenced by comorbid conditions or confounds (including HIV-related opportunistic CNS conditions, or unrelated developmental, psychiatric, or neuromedical confounds).

Table E-2 provides an algorithm, or an outline of basic criteria, for classifying the three neurocognitive disorders. Separate columns specify similar criteria (levels of impairment) that can be met using formal neuropsychological testing vs clinical mental status testing. In order to reliably use the algorithm in table E-2, additional operational definitions and guidelines are needed regarding establishing neurocognitive impairment, functional decline, and confounds. This additional guidance about methods of assessment and interpretation is provided in tables E-2 and E-4, and related text below.

To facilitate reliable use of this algorithm, we suggest specific examples of standardized assessments that can be used to establish the various criteria in table E-2. It is recognized, however, that in many resource-limited settings, standardized neurobehavioral examination procedures and other diagnostic technologies (e.g., MRI scanning) are not yet available. In particular, the neuropsychological tests and functional assessments listed in table E-3 may not have been validated in the languages of many countries, or appropriate normative standards may be lacking. In such cases the algorithm may still be followed in principle, using clinical assessments and clinical judgment aimed at establishing the same criteria. Ideally, these clinical methods themselves will become increasingly standardized, so that results can be compared across patients and across clinicians. Table E-4 provides descriptions of comorbid conditions and their grading, but is not designed to be exhaustive. Rather it is intended to assist clinicians in grading these particular conditions in patients who otherwise meet criteria for HAND. They also provide conceptual guidelines that should be more broadly applicable to a range of comorbid conditions, and hopefully will promote more reliable classification of confounds in general.

\section{Neurocognitive impairment}

If neuropsychological (NP) testing is available, this testing should cover multiple ability domains (see below). Test results on at least two of these domains must be abnormal in order for the patient to be classified overall as having NP impairment. Impairment on individual tests would require a performance that is more than one SD below the mean of a demographically comparable HIV seronegative group, or greater than one SD below the normative mean using published norms that are demographically adjusted (for age, education, gender, and/or ethnicity, as appropriate for the test). The importance of addressing appropriate norms was described above. Ideally, the examination would include tests of the following ability domains (if possible, with at least two test measures per 
domain): verbal/language; attention/working memory; abstraction/executive; memory (learning; recall); speed of information processing; sensory-perceptual; motor skills. Neurocognitive impairment requires that at least one of the tested ability deficits be primarily cognitive in nature (i.e., impairment that is limited solely to motor and sensoryperceptual functions would not qualify). Classification of moderate or greater overall NP impairment (a criterion for $\mathrm{HAD}$ ) requires performances on two ability domains that are greater than 2 SD below the normative mean; alternatively, the patient could score greater than $2.5 \mathrm{SD}$ below normative expectations (an operational definition for moderate to severe impairment) for one domain and greater than one SD below expectations for another.

Further details regarding procedures for reliably classifying NP impairment in HIV-infected adults, involving multiple ability domains and multiple test measures within each domain, can be found in Woods et al. ${ }^{11}$

Examples of published NP tests within the above-mentioned ability domains along with selected references are provided in table E-3. This list is not intended to be exhaustive, and primarily includes tests that have been standardized in Western countries (particularly the United States) and used with HIV-infected populations there. Many of these tests are being adapted for use in other regions (e.g., in Asia, Africa, and South America). However, population-specific normative standards and evidence of cross-cultural validity are needed before such tests can be used to confidently diagnose individual patients who have substantially different linguistic, cultural, and/or educational backgrounds than people in the original test standardization samples.

If NP testing is not available, presence of cognitive impairment involving two or more ability domains may be detected by standardized mental status examinations (MSEs), using demographically appropriate normative cutoffs if available. For example, in the United States and other Western countries, mild cognitive impairment might be inferred if a young or middle aged adult ( $<60$ years old) with at least 12 years of education achieves scores of 25 to 26 on the Folstein et al. ${ }^{12}$ Mini-Mental State Examination; a score less than 25 would signify moderate (or worse) impairment (cutoffs estimated from data provided in Crum et al. ${ }^{13}$ ). Other MSEs that might be used include the HIV Dementia Scale, ${ }^{14}$ the International HIV Dementia Scale, ${ }^{15}$ and the Mattis Dementia Rating Scale. ${ }^{16}$ Ideally, in considering impairment, the patient should also give evidence of involvement of at least two different aspects of cognition. This is consistent with the requirements for when neuropsychological testing is available.

It is not possible to provide a complete listing here of items on MSEs that may demonstrate impairments of the cognitive domains mentioned above. However, some examples from the Mini-Mental State Examination are as follows: Registration = verbal learning, Attention and Concentration (both serial 7s and spelling "world" backwards) = attention/working memory, Recall $=$ verbal memory, Language $=$ language skills, and Copy Design $=$ spatial skills. For the HIV Dementia Scale, Anti-saccadic Errors = Attention, Psychomotor Speed = speed of information processing, Memory Recall $=$ verbal memory, and Construction $=$ perceptual spatial skills. Similarly, for the International HIV Dementia Scale, Psychomotor Speed (rapid motor sequencing) = either executive functions or speed of information processing, Memory-Recall $=$ verbal memory, and Motor Speed $=$ motor . 
The working group recognized that extrapyramidal abnormalities (such as rigidity, bradykinesia, or hypomimia) and psychiatric features (such as apathy, personality change, irritability, or disinhibition) occur commonly in this context. We decided not to include these in the proposed classification criteria because such symptoms often are difficult to establish as a result of HIV infection (as opposed to representing pre-existing or comorbid phenomena). Furthermore, there is insufficient evidence that these symptoms are reliably associated with neurocognitive impairments or (nonbehavioral) indications of CNS involvement of HIV-1 (e.g., neuropathology, neuroimaging abnormalities, CSF viral load, biomarkers of inflammatory processes).

Future research should assess reliability of diagnostic classifications based upon clinical methods vs more formal testing methods. Validity of the diagnoses may be studied by examining relationships with disease history and outcomes, imaging and biomarker evidence of CNS involvement, and neuropathologic criteria.

\section{Functional decline}

Acquired impairment of everyday functioning can be assessed by self report or report of a knowledgeable informant (family member, close friend, caregiver), or by objective assessment of the patient's ability to perform cognitively related instrumental ADLs (IADLs) such as financial management and medication management. Both the report-based information and objective functional assessments should be obtained using standardized instruments, and ideally these instruments would have normative guidelines that are appropriate to the patient being examined (i.e., ideally norms would be available for the patient's country and demographic peer group). Available questionnaires assess the frequency with which patients experience difficulties with various aspects of cognition in their everyday lives, and increased dependence upon others in their IADLs. ${ }^{17}$ Also, standardized tasks are available to objectively assess abilities to, for example, manage medications, manage finances, shop, cook, perform job-related activities, and drive an automobile. ${ }^{17-22}$

Objective, laboratory-based assessment of functional impairment adds time to the diagnostic evaluation and may require specialized test materials or equipment.

Also, such assessment is not needed in all cases. Objective assessment of functional skills is likely to be most informative when a patient meets other criteria for an HIV-related neurocognitive disorder, but denies being aware of any change in everyday functioning.

Mild functional decline requires at least two of the following that are not readily attributable to comorbid conditions in the judgment of the examiner:

1. Self report or other report of some increased assistance with at least two IADLs such as medication management, financial management, shopping, meal preparation, light housekeeping, laundry, driving, use of public transportation, maintaining personal schedules, understanding media events, and child care. (More IADLs could be considered as appropriate to the individual.) 
2. Patient is unable to perform some aspects of a previous job. This is not due to medical symptoms.

3. Although patient may maintain employment and/or full IADL independence, he or she reports less efficiency, reduced productivity, more errors in performing tasks, more difficulty meeting expectations, or greater effort expended performing the same activities.

4. In the absence of significant depression (e.g., Beck Depression Inventory $\geq 17^{23}$ ), ${ }^{1}$ which may bias reporting of symptoms, patient reports that he or she is experiencing increased difficulty with $\geq 2$ aspects of cognition in daily life. These may include difficulties with memory for recent events (people, conversations, names, commitments, where things are placed), understanding conversations or reading materials, word finding, planning activities, problem solving, concentrating, thinking clearly or logically, finding his or her way about, calculating, or following directions or instructions. Reports of these difficulties also may be obtained from a knowledgeable informant. (Instruments to assess depression that allow one to separate out items concerning somatic symptoms from those describing depressed mood are preferred for this purpose, as somatic symptoms associated with depression may also be caused by HIV itself.)

5. If performance-based, standardized functional tests are administered, patient scores $>1 \mathrm{SD}$ below an appropriate normative mean on at least one such task.

Major functional decline requires two or more of the following that are not readily attributable to medical or other comorbid conditions in the judgment of the examiner:

1. Patient is unable to maintain former employment and this is not due to systemic illness or other factors not related to cognitive impairment (e.g., healthcare coverage being dependent upon disability status).

2. Patient requires substantially greater assistance (or is dependent) with more than two IADLs, as listed above.

3. Patient or a knowledgeable informant reports that he or she experiences/shows significantly greater difficulty with $\_4$ aspects of cognition, as listed above. However, self report is not sufficient (would need confirmation by another informant) if patient is significantly depressed (e.g., BDI $\geq 17$ ).

4. If performance-based, standardized functional tasks are administered, patient scores $>2$ SD below an appropriate normative mean on at least one such task, or $>1 \mathrm{SD}$ below the mean on at least two tasks.

Questionnaires and performance-based tests for measuring functional decline have been standardized in Western countries, and many have been used in published studies of HIVinfected groups. As is the case with NP testing, measures of everyday functioning (and available normative standards for them) cannot be assumed to be valid across populations having substantially different linguistic, cultural, and educational backgrounds. 
ADLs themselves may be different in different cultures (e.g., reflecting differences in common modes of transportation, shopping, and financial transactions). Thus, compared to NP testing, which is intended to measure more fundamental human abilities, measures of functional decline may require even more adaptation for cross-cultural use. Before they are selected for use in a new (different) population, IADL tests and questionnaires should be carefully screened for relevance to the everyday lives of the people involved.

There is an additional consideration for cross-cultural use of the current algorithm: HIV-1 related changes in cognitive ability may be much less likely to cause meaningful functional decline in cultures or living situations that pose fewer demands for such abilities. If more severe cognitive changes are needed to cause meaningful functional declines in some settings, the prevalence of symptomatic neurocognitive disorders (MND and HAD) may be significantly lower in those settings. This could have unfortunate consequences if excessive numbers of patients in these settings who do have HIV-1 associated brain dysfunction fail to be identified as such. The current nomenclature's addition of an asymptomatic category of neurocognitive impairment may help prevent such cases from being missed.

\section{Other sources of NP impairment and functional limitations (comorbid conditions)}

The algorithm for diagnosing the three HIV-1-associated neurocognitive disorders (table E-2) requires the clinician to make the judgment that these disorders reflect the effects of HIV-1 infection on the brain. As described above, in Section 1, the observed NP impairments and functional limitations cannot be explained on the bases of CNS opportunistic diseases, medications with CNS effects, or developmental or acquired conditions unrelated to HIV-1. The number of such potential confounds, in all of their manifestations, is exceedingly large, so it would be impossible to specify how to rate the importance of each of them. A second level of comorbid condition is considered to be a contributing condition. This means that the condition probably has had some substantive contribution to the observed NP impairments or functional limitations, but that the effect of HIV-1 also is considered to be significant. For example, in the case of a serious developmental disorder or previous traumatic brain injury, subsequent progression of NP impairment or functional decline may have been documented within the context of HIV-1 infection. This would demonstrate a significant role for HIV-1. It is emphasized that the presence of a contributing condition does not preclude the diagnosis of an HIV-1-associated neurocognitive disorder, although the severity of the HIV-1 component may be more difficult to ascertain.

\section{GLOSSARY}
AAN
American Academy of Neurology
ADLs
activities of daily living
ANI
asymptomatic neurocognitive impairment
HAART
highly active antiretroviral treatment
HAD
HIV-associated dementia 


$\begin{array}{ll}\text { HAND } & \text { HIV-associated neurocognitive disorders } \\ \text { HNRC } & \text { HIV Neurobehavioral Research Center } \\ \text { IADLs } & \text { instrumental ADLs } \\ \text { MCMD } & \text { minor cognitive motor disorder } \\ \text { MND } & \text { HIV-associated mild neurocognitive disorder } \\ \text { MSE } & \text { mental status examination }\end{array}$

\section{References}

1. Janssen RS, Cornblath DR, Epstein LG, et al. Nomenclature and research case definitions for neurological manifestations of human immunodeficiency virus type-1 (HIV-1) infection. Report of a Working Group of the American Academy of Neurology AIDS Task Force. Neurology. 1991; 41:778-785. [PubMed: 2046917]

2. Cherner M, Cysique L, Heaton RK, et al. Neuropathologic confirmation of definitional criteria for human immunodeficiency virus-associated neurocognitive disorders. J Neurovirol. 2007; 13:23-28. [PubMed: 17454445]

3. Cherner M, Masliah E, Ellis RJ, et al. Neurocognitive dysfunction predicts postmortem findings of HIV encephalitis. Neurology. 2002; 59:1563-1567. [PubMed: 12451198]

4. Wojna V, Skolasky RL, Hechavarria R, et al. Prevalence of human immunodeficiency virusassociated cognitive impairment in a group of Hispanic women at risk for neurological impairment. J Neurovirol. 2006; 12:356-364. [PubMed: 17065128]

5. Arendt G, Hefter H, Hilperath F, von Giesen HJ, Strohmeyer G, Freund HJ. Motor analysis predicts progression in HIV-associated brain disease. J Neurol Sci. 1994; 123:180-185. [PubMed: 8064312]

6. Brew BJ. Evidence for a change in AIDS dementia complex in the era of highly active antiretroviral therapy and the possibility of new forms of AIDS dementia complex. AIDS. 2004; 18(suppl 1):S75S78. [PubMed: 15075501]

7. Dore GJ, Correll PK, Li Y, Kaldor JM, Cooper DA, Brew BJ. Changes to AIDS dementia complex in the era of highly active antiretroviral therapy (in process citation). AIDS. 1999; 13:1249-1253. [PubMed: 10416530]

8. Dore GJ, McDonald A, Li Y, Kaldor JM, Brew BJ. Marked improvement in survival following AIDS dementia complex in the era of highly active antiretroviral therapy. AIDS. 2003; 17:15391545. [PubMed: 12824792]

9. Dore GJ, McDonald A, Li Y, Kaldor JM, Brew BJ. Marked improvement in survival following AIDS dementia complex in the era of highly active antiretroviral therapy. AIDS. 2003; 17:15391545. [PubMed: 12824792]

10. Brew BJ. Evidence for a change in AIDS dementia complex in the era of highly active antiretroviral therapy and the possibility of new forms of AIDS dementia complex. AIDS. 2004; 18(suppl 1):S75-S78. [PubMed: 15075501]

11. Woods SP, Rippeth JD, Frol AB, et al. Interrater reliability of clinical ratings and neurocognitive diagnoses in HIV. J Clin Exp Neuropsychol. 2004; 26:759-778. [PubMed: 15370374]

12. Folstein MF, Folstein SE, McHugh PR. Mini-Mental State: a practical method for grading the cognitive state of patients for the clinician. J Psychiatr Res. 1975; 12:189-198. [PubMed: 1202204]

13. Crum RM, Anthony JC, Bassett SS, Folstein MF. Population-based norms for the Mini-Mental State Examination by age and educational level. JAMA. 1993; 269:2386-2391. [PubMed: 8479064]

14. Power C, Selnes OA, Grim JA, McArthur JC. The HIV Dementia Scale: a rapid screening test. J AIDS. 1995; 8:273-278. 
15. Sacktor NC, Wong M, Nakasujja N, et al. The International HIV Dementia Scale: a new rapid screening test for HIV dementia. AIDS. 2005; 19:1367-1374. [PubMed: 16103767]

16. Mattis, S. Dementia Rating Scale (DRS). Odessa, FL: Psychological Assessment Resources, Inc; 1988.

17. Heaton RK, Marcotte TD, Mindt MR, et al. The impact of HIV-associated neuropsychological impairment on everyday functioning. J Int Neuropsychol Soc. 2004; 10:317-331. [PubMed: 15147590]

18. Albert SM, Weber C, Todak G, et al. An observed performance test of medication management ability in HIV: relation to neuropsychological status and adherence outcomes. AIDS and Behavior. 1999; 3:121-128.

19. Lowenstein, DA.; Bates, BC. Manual for administration and scoring the Direct Assessment of Functional Status scale in older adults (DAFS). Miami Beach, FL: Mt Sinai Medical Center; 1992.

20. Marcotte TD, Wolfson T, Rosenthal TJ, et al. A multimodal assessment of driving performance in HIV infection. Neurology. 2004; 63:1417-1422. [PubMed: 15505158]

21. Patterson TL, Lacro J, McKibbin CL, Moscona S, Hughs T, Jeste DV. Medication management ability assessment: results from a performance-based measure in older outpatients with schizophrenia. J Clin Psychopharmacol. 2002; 22:11-19. [PubMed: 11799337]

22. Patterson TL, Goldman S, McKibbin CL, Hughs T, Jeste DV. UCSD Performance-Based Skills Assessment: development of a new measure of everyday functioning for severely mentally ill adults. Schizophr Bull. 2001; 27:235-245. [PubMed: 11354591]

23. Kalichman SC, Rompa D, Cage M. Distinguishing between overlapping somatic symptoms of depression and HIV disease in people living with HIV-AIDS. J Nerv Ment Dis. 2000; 188:662670. [PubMed: 11048815]

24. Ramachandran, VS., editor. Encyclopedia of the Human Brain. San Diego: Academic Press; 2002. Grant I The neurocognitive complications of HIV infection; p. 475-489. 


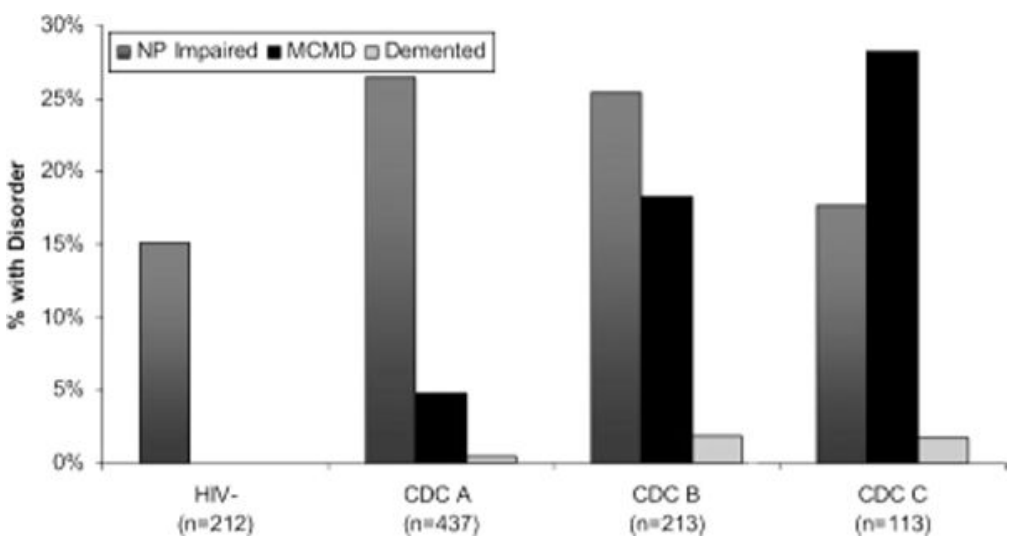

Figure 1.

Prevalence of neurocognitive disorders by stage of HIV disease 


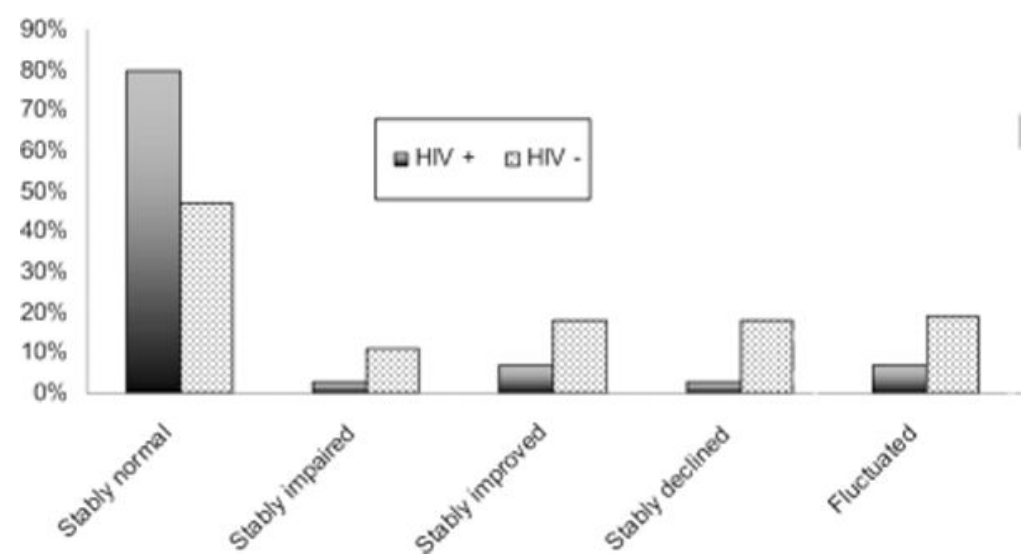

Figure 2.

Neuropsychological course for neurocognitive states of HIV+ $(n=534)$ vs HIV- subjects (n $=30$ ) 


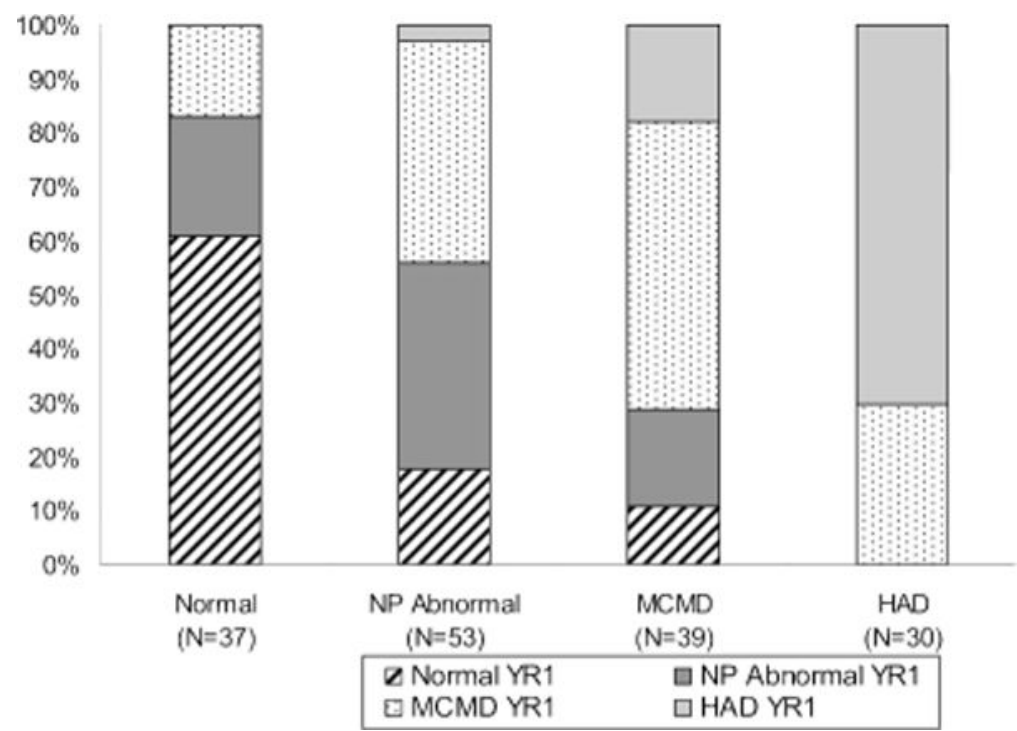

Figure 3.

Hawaii Aging with HIV cohort: Diagnostic transitions from baseline to year 1 


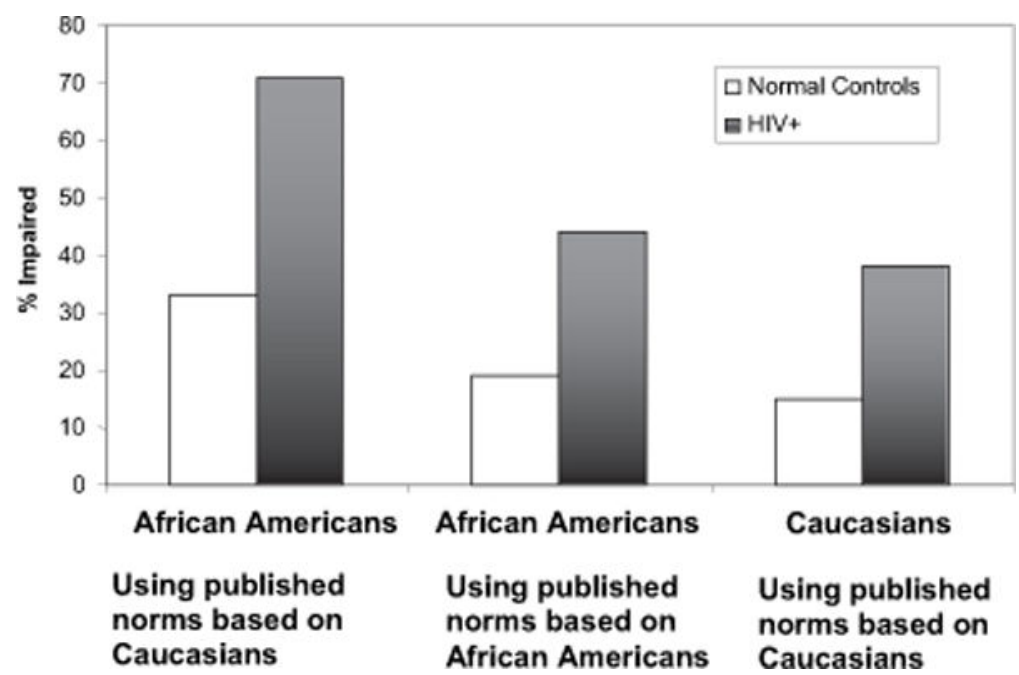

Figure 4.

Results of using different normative data sets to identify neuropsychological impairment in African American subjects 


\section{Table}

\section{Revised research criteria for HIV-associated neurocognitive disorders (HAND) (modified from HIV Neurobehavioral Research Center criteria ${ }^{24}$ )}

HIV-associated asymptomatic neurocognitive impairment (ANI)*

1 Acquired impairment in cognitive functioning, involving at least two ability domains, documented by performance of at least 1.0 $\mathrm{SD}$ below the mean for age-education-appropriate norms on standardized neuropsychological tests. The neuropsychological assessment must survey at least the following abilities: verbal/language; attention/working memory; abstraction/executive; memory (learning; recall); speed of information processing; sensory-perceptual, motor skills.

2 The cognitive impairment does not interfere with everyday functioning.

3 The cognitive impairment does not meet criteria for delirium or dementia.

4 There is no evidence of another preexisting cause for the ANI. ${ }^{\dagger}$

*If there is a prior diagnosis of ANI, but currently the individual does not meet criteria, the diagnosis of ANI in remission can be made.

If the individual with suspected ANI also satisfies criteria for a major depressive episode or substance dependence, the diagnosis of ANI should be deferred to a subsequent examination conducted at a time when the major depression has remitted or at least 1 month after cessation of substance use.

HIV-1-associated mild neurocognitive disorder (MND)*

1 Acquired impairment in cognitive functioning, involving at least two ability domains, documented by performance of at least 1.0 $\mathrm{SD}$ below the mean for age-education-appropriate norms on standardized neuropsychological tests. The neuropsychological assessment must survey at least the following abilities: verbal/language; attention/working memory; abstraction/executive; memory (learning; recall); speed of information processing; sensory-perceptual, motor skills.

Typically, this would correspond to an MSK scale stage of 0.5 to 1.0

2 The cognitive impairment produces at least mild interference in daily functioning (at least one of the following):

a. Self-report of reduced mental acuity, inefficiency in work, homemaking, or social functioning.

b. Observation by knowledgeable others that the individual has undergone at least mild decline in mental acuity with resultant inefficiency in work, homemaking, or social functioning.

3 The cognitive impairment does not meet criteria for delirium or dementia.

4 There is no evidence of another preexisting cause for the MND. ${ }^{\dagger}$

*If there is a prior diagnosis of MND, but currently the individual does not meet criteria, the diagnosis of MND in remission can be made.

II the individual with suspected MND also satisfies criteria for a severe episode of major depression with significant functional limitations or psychotic features, or substance dependence, the diagnosis of MND should be deferred to a subsequent examination conducted at a time when the major depression has remitted or at least 1 month after cessation of substance use.

HIV-1-associated dementia (HAD)*

1 Marked acquired impairment in cognitive functioning, involving at least two ability domains; typically the impairment is in multiple domains, especially in learning of new information, slowed information processing, and defective attention/concentration. The cognitive impairment must be ascertained by neuropsychological testing with at least two domains 2 SD or greater than demographically corrected means. (Note that where neuropsychological testing is not available, standard neurological evaluation and simple bedside testing may be used, but this should be done as indicated in algorithm; see below).

Typically, this would correspond to an MSK scale stage of 2.0 or greater.

2 The cognitive impairment produces marked interference with day-to-day functioning (work, home life, social activities)

3 The pattern of cognitive impairment does not meet criteria for delirium (e.g., clouding of consciousness is not a prominent feature); or, if delirium is present, criteria for dementia need to have been met on a prior examination when delirium was not present.

4 There is no evidence of another, preexisting cause for the dementia (e.g., other CNS infection, CNS neoplasm, cerebrovascular disease, preexisting neurologic disease, or severe substance abuse compatible with CNS disorder). ${ }^{\dagger}$

*If there is a prior diagnosis of HAD, but currently the individual does not meet criteria, the diagnosis of HAD in remission can be made

$\dagger$ If the individual with suspected HAD also satisfies criteria for a severe episode of major depression with significant functional limitations or psychotic features, or substance dependence, the diagnosis of HAD should be deferred to a subsequent examination conducted at a time when the major depression has remitted or at least 1 month has elapsed following cessation of substance use. Note that the consensus was that even 
when major depression and HAD occurred together, there is little evidence that pseudodementia exists and the cognitive deficits do not generally improve with treatment of depression. 